\section{Autophagie et micro-organismes}

Delphine Le Roux, Florence Niedergang

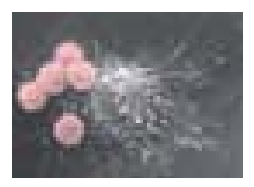

Équipe Phagocytose et invasion bactérienne, Institut Cochin, CNRS UMR8104, Inserm U1016, Université Paris Descartes, 22, rue Méchain, 75014 Paris, France. delphine.le-roux@inserm.fr florence.niedergang@inserm.fr
«Autophagie et micro-organismes», c'est le thème d'actualité qui a été choisi par le nouveau département de Biologie cellulaire et interactions hôtes-pathogènes de I'Institut Cochin pour son premier mini-symposium qui s'est tenu le 30 novembre 2009. Les organisateurs, Florence Niedergang, Alexandre Benmerah et Maryline Bajolle, avaient réuni une palette d'orateurs de renom dans le domaine et nous souhaitons résumer ici leurs interventions.

\section{Qu'est ce que l'autophagie?}

La première présentation a été faite par Vojo Deretic (University of New Mexico Health Sciences Center, États-Unis) qui a magistralement introduit le sujet et nous a présenté une revue des connaissances récentes sur l'autophagie. Le terme d' «autophagie » a été créé par Christian De Duve il y a plus de cinquante ans. Ce processus amène la cellule à s'autodigérer, à dégrader une partie de son cytoplasme par ses propres lysosomes. Induite en cas de privation ou de stress, l'autophagie sert à recycler les acides aminés pour maintenir en activité les mécanismes essentiels à la survie cellulaire et à éliminer les organites endommagés ou les agrégats de protéines mal formées. Les étapes de formation des autophagosomes commencent à être bien décrites (voir [1] et Figure 1A). Un des marqueurs de l'autophagie est la protéine LC3, répartie normalement de façon diffuse dans le cytoplasme et qui, une fois clivée, se distribue sous une forme ponctuée spécifique et caractéristique de la mise en place du processus autophagique (Figure l). Grâce à I'utilisation de souris transgéniques exprimant la protéine LC3 couplée à la GFP (green fluorescent protein), Vojo Deretic a montré que l'autophagie pouvait aussi servir à éliminer les pathogènes intracellulaires comme
Mycobacterium tuberculosis (voir [2] et Figure 1). Plus récemment, il s'est intéressé aux relations entre autophagie et infection des macrophages par le virus de l'immunodéficience humaine (VIH-1) [3]. En effet, les virus colocalisent avec des marqueurs d'autophagosome dans les macrophages et l'induction pharmacologique de l'autophagie augmente la production virale. De plus, son équipe a mis en évidence que la protéine Nef du VIH-l bloque la maturation de l'autophagosome mais pas l'initiation de sa formation, permettant ainsi le développement du virus. En effet, Nef est capable d'inhiber la destruction du virus par autophagie en interagissant avec la protéine Bécline-1, important régulateur du processus (Figure 1). Ainsi, le virus semble utiliser les premières étapes de formation des autophagosomes à son profit et inhiber les étapes tardives grâce à la protéine Nef.

\section{Autophagie et infection bactérienne : l'exemple de Shigella flexneri}

Frank Lafont (Institut Pasteur de Lille) nous a ensuite présenté ses récents travaux sur les liens entre autophagie et infection par les bactéries Shigella flexneri et Yersinia pseudotuberculosis. Shigella est une bactérie entéro-invasive qui, une fois qu'elle a pénétré dans les cellules épithéliales, lyse rapidement la membrane de la vacuole dans laquelle elle se trouve et devient cytosolique. Les travaux de Frank Lafont ont montré que les protéines associées aux « restes 》 de membrane vacuolaire sont polyubiquitinylées, ce qui permet ainsi leur élimination par autophagie [4]. De plus, l'autophagie induite pour éliminer ces morceaux de membrane régule la réaction inflammatoire déclenchée par l'infection par S. flexneri. En effet, la production d'interleukine- 8 (IL-8) augmente lorsque $S$. flexneri infecte des cellu- 


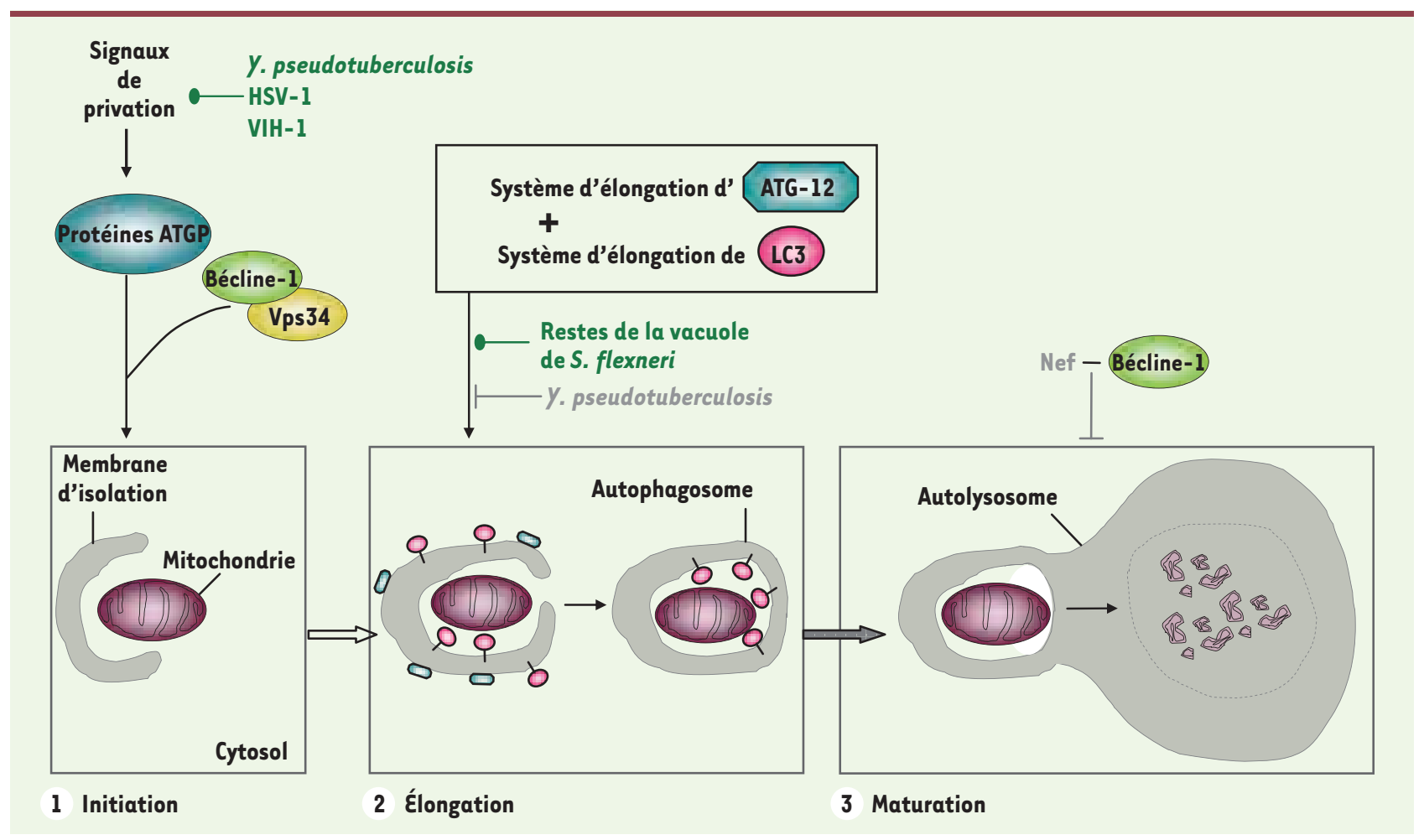

Figure 1. Les différentes étapes de la formation d'un autophagosome et les protéines impliquées. L'autophagie comprend 3 phases distinctes: I'initiation, l'élongation et la maturation. La première phase (1) est initiée par la cellule en souffrance, les protéines ATG (autophagy-related proteins) sont activées et la membrane isolant l'organite endommagé ou le pathogène commence à se former. La deuxième phase (2) dite d'élongation fait intervenir le complexe ATG12 (formé des protéines ATG12, 5 et 16) ainsi que la protéine LC-3, qui, une fois clivée, se localise dans l'autophagosome. Dans la troisième étape (3) il y a fusion de l'autophagosome avec les lysosomes permettant ainsi la dégradation de l'organite ou du pathogène. Nous avons également indiqué ici comment certains pathogènes cités dans cette revue peuvent moduler cette voie à leur avantage (activateurs, inhibiteurs).

les épithéliales dépourvues de certaines protéines importantes pour l'autophagie (Atg5 ou p62, une protéine requise pour l'élimination des protéines polyubiquitinylées par autophagie). Les bactéries Yersinia pseudotuberculosis, lorsqu'elles pénètrent dans les macrophages et y survivent, activent le mécanisme d'autophagie, mais perturbent le devenir de l'autophagosome, empêchant son acidification et la dégradation protéique [5]. De plus, l'autophagie est nécessaire à la réplication des bactéries. Enfin, elle est nécessaire à l'induction de la pyroptose, une mort cellulaire inflammatoire qui dépend de la caspase-1 (contrairement à l'apoptose, l'autre mort cellulaire faisant intervenir les caspases, mais non inflammatoire). Ces résultats montrent comment l'autophagie régule la mort cellulaire lors d'une infection.

\section{Autophagie et parasites : l'exemple de Theileria, agent de la theilériose}

Très peu d'études de la littérature se sont focalisées jusqu'à maintenant sur l'autophagie dans le cadre des maladies à protozoaires parasites, dans les parasites eux-mêmes ou dans les cellules infectées par des parasites. Gordon Langsley (Institut Cochin, Paris) nous a présenté ses derniers résultats sur Theileria, agent de la theilériose, maladie hématologique proliférative transmise par les tiques et qui affecte les bovins. De manière intéressante, il a observé que l'administration d'un traitement médicamenteux antiparasitaire entraînait la formation, dans les lymphocytes B infectés, de vacuoles intracellulaires possédant une double membrane et dont la morphologie rappelait celle d'un autophagosome. Des analyses par microscopie à fluorescence ont montré que lorsque la mort des parasites est induite par des drogues, le parasite mort est reconnu par autophagie et éliminé, alors que le parasite vivant ne l'est pas. Ceci suggère que le parasite vivant bloque une étape dans le déclenchement du processus d'autophagie. 0 n peut faire l'hypothèse que le parasite intracellulaire, en bloquant l'autophagie, diminue la présentation antigénique et l'activation des lymphocytes $T$ cytotoxiques et échappe ainsi à la réponse immunitaire 
efficace. Ces travaux novateurs seront sans doute le prélude à de nombreuses études excitantes sur les liens entre l'autophagie et les parasites.

\section{Autophagie et infections virales : l'exemple du VIH}

Par des études poussées de microscopie électronique, Philippe Benaroch (Institut Curie, Paris) et son équipe ont observé que les virions du VIH s'assemblent dans des compartiments intracellulaires aux caractéristiques inhabituelles. En effet, de manière surprenante, alors que les macrophages ont une grande capacité de dégradation d'antigènes, les compartiments contenant les virions sont peu ou pas acidifiés [6]. Ces compartiments contiennent certaines tétraspanines ${ }^{1}$ et des protéines du complexe ESCRT (endosomal sorting complex required for transport) mais leur identification (nature, fonction) ainsi que leur relation aux voies d'endocytose et de sécrétion restent encore à définir. Récemment, Philippe Benaroch et son équipe ont voulu savoir si l'induction d'autophagie dans des cellules infectées entraînait la destruction des stocks intracellulaires de virions. En utilisant la lignée de monocytes humains THPl exprimant de façon ectopique la protéine LC3GFP, ils ont montré que le traitement à la rapamycine (un inducteur de l'autophagie) diminuait à la fois la quantité de virions produits et leur pouvoir infectieux. Ces résultats ont été discutés à la lumière des données présentées par Vojo Deretic plus tôt dans la matinée. II est en effet possible que la rapamycine outrepasse le blocage de l'autophagie dû à Nef. L'équipe de Philippe Benaroch s'attache actuellement à disséquer les mécanismes moléculaires sous-jacents à ces observations et ceux qui permettent la sécrétion des virions.

\section{Autophagie et présentation antigénique}

Le mini-symposium s'est clôturé par une présentation de Michel Desjardins (Université de Montréal, Canada) sur les implications de l'autophagie pour la présentation antigénique. Classiquement, les antigènes viraux sont présentés par les molécules du CMH (complexe majeur d'histocompatibilité) de classe I permettant l'activation de lymphocytes $\operatorname{CD} 8^{+}$dès six heures après l'infection des macrophages. L'équipe de

${ }^{1}$ Les tétraspanines (CD9, CD63, CD81 ou CD151) sont des glycoprotéines de surface de 200 à 300 acides aminés, et se composent de quatre régions hydrophobes probablement transmembranaires. Alors que la fonction moléculaire n'est pas encore déterminée et qu'aucun ligand physiologique n'a pu être décrit pour ces molécules, elles sont impliquées dans une multitude d'interactions à la surface cellulaire, suggérant que leur fonction est en relation avec la formation de complexes multimoléculaires (Eric Rubinstein).
Michel Desjardins a observé une potentialisation de cette activation en fonction du temps avec un maximum douze heures après infection de macrophages par le virus de l'herpès (HSV-1). De manière intéressante, cette activation à douze heures est sensible au traitement par la bafilomycine $A$, une drogue qui inhibe la pompe à protons vacuolaire et empêche ainsi l'acidification des endosomes, alors que l'activation précoce (six à huit heures post-infection) des lymphocytes T CD8 ${ }^{+}$n'est pas affectée. Ces résultats suggéraient l'existence de deux voies de présentation des antigènes viraux par les macrophages infectés: la première «classique», cytosolique et dépendante du protéasome, et une seconde impliquant un compartiment intracellulaire de dégradation acide qui augmente considérablement la présentation antigénique. Dans les macrophages infectés par le HSV-1, la protéine LC3 est clivée et s'accumule six heures après l'infection, alors qu'elle est absente dans les macrophages non infectés. Ceci indique clairement qu'un processus autophagique est initié par l'infection et qu'il est concomitant de la deuxième phase d'activation des lymphocytes $T \operatorname{CD}^{+}$. Cette équipe de chercheurs s'est donc attachée à établir un lien entre ces deux observations et a mis en évidence le fait que l'induction de l'autophagie dans les macrophages participe à une meilleure présentation des antigènes viraux par les molécules du CMH de classe I et par conséquent à une augmentation de l'activation des lymphocytes T CD8 ${ }^{+}$. Ils ont également mis en évidence par microscopie électronique des autophagosomes classiques contenant les particules virales et identifiés par une double membrane, qui rappellent le phénotype décrit plus haut dans les cellules infectées traitées par la rapamycine, et surtout, ils ont observé un autre type d'autophagosome: à quatre membranes. Ce nouveau type d'autophagosome émerge du noyau, n'est visible que dans les cellules infectées et peut fusionner avec les organites lytiques permettant la dégradation des particules virales [7] (voir aussi le texte récent de M. Desjardins dans médecine/sciences [8]). Ainsi dans ce contexte, l'autophagie sert à éliminer le pathogène, notamment en induisant une meilleure destruction intracellulaire et en potentialisant la réponse immunitaire.

\section{En conclusion}

L'autophagie est induite à la suite d'un stress de privation des cellules ou, comme le montrent les travaux des différents orateurs intervenant dans ce mini-symposium, une infection intracellulaire. Ces différents séminaires nous ont montré que les pathogènes peuvent détourner ce processus à leur avantage, comme Y. pseudotuberculosis ou le VIH-1. Cependant, il est important de noter que la cellule se sert également de cette voie pour éliminer les microorganismes intracellulaires comme le virus HSV-1 (Figure 1). Ainsi, cette dualité de l'autophagie, dont ce minisymposium a révélé toute la complexité, illustre bien l'importance grandissante de cette voie de dégradation dans de nombreux processus, comme la réponse immunitaire, mais aussi le développement ou la tumorigenèse. $\diamond$

«Autophagy and microorganisms 》:

hotspots of a mini-symposium 


\section{CONFLIT D'INTÉRÊTS}

Les auteurs déclarent n'avoir aucun conflit d'intérêts concernant les données publiées dans cet article.

\section{RÉFÉRENCES}

1. Levine B, Deretic V. Unveiling the roles of autophagy in innate and adaptive immunity. Nat Rev Immunol $2007 ; 7: 767-77$.

2. Gutierrez MG, Master SS, Singh SB, et al. Autophagy is a defense mechanism inhibiting BCG and Mycobacterium tuberculosis survival in infected macrophages. Cell $2004 ; 119: 753-66$

3. Kyei GB, Dinkins C, Davis AS, et al. Autophagy pathway intersects with HIV-1 biosynthesis and regulates viral yields in macrophages. J Cell Biol $2009 ; 186: 255-68$.

4. Dupont N, Lacas-Gervais S, Bertout J, et al. Shigella phagocytic vacuolar membrane remnants participate in the cellular response to pathogen invasion and are regulated by autophagy. Cell Host Microb $2009 ; 6$ : 137-49.
5. Moreau K, Lacas-Gervais S, Fujita N, et al. Autophagosome can support Yersinia pseudotuberculosis replication in macrophages. Cell Microbiol 2010 (sous presse)

6. Jouve M, Sol-Foulon N, Watson S, et al. HIV-1 buds and accumulates in «nonacidic » endosomes of macrophages. Cell Host Microb 2007 $2: 85-95$.

7. English L, Chemali M, Duron J, et al. Autophagy enhances the presentation of endogenous viral antigens on $\mathrm{MHC}$ class I molecules during HSV-l infection. Nat Immunol 2009 : 10:480-7.

8. English L, Chemali M, Desjardins M. Contribution de l'autophagie dan l'apprêtement d'antigènes viraux endogènes. Med Sci (Paris) 2009 ; $25: 906-9$

\section{TIRÉS À PART}

F. Niedergang
Promega

Tour

et la Bioluminescence

\section{L'Analyse cellulaire et a Bioluminescence}

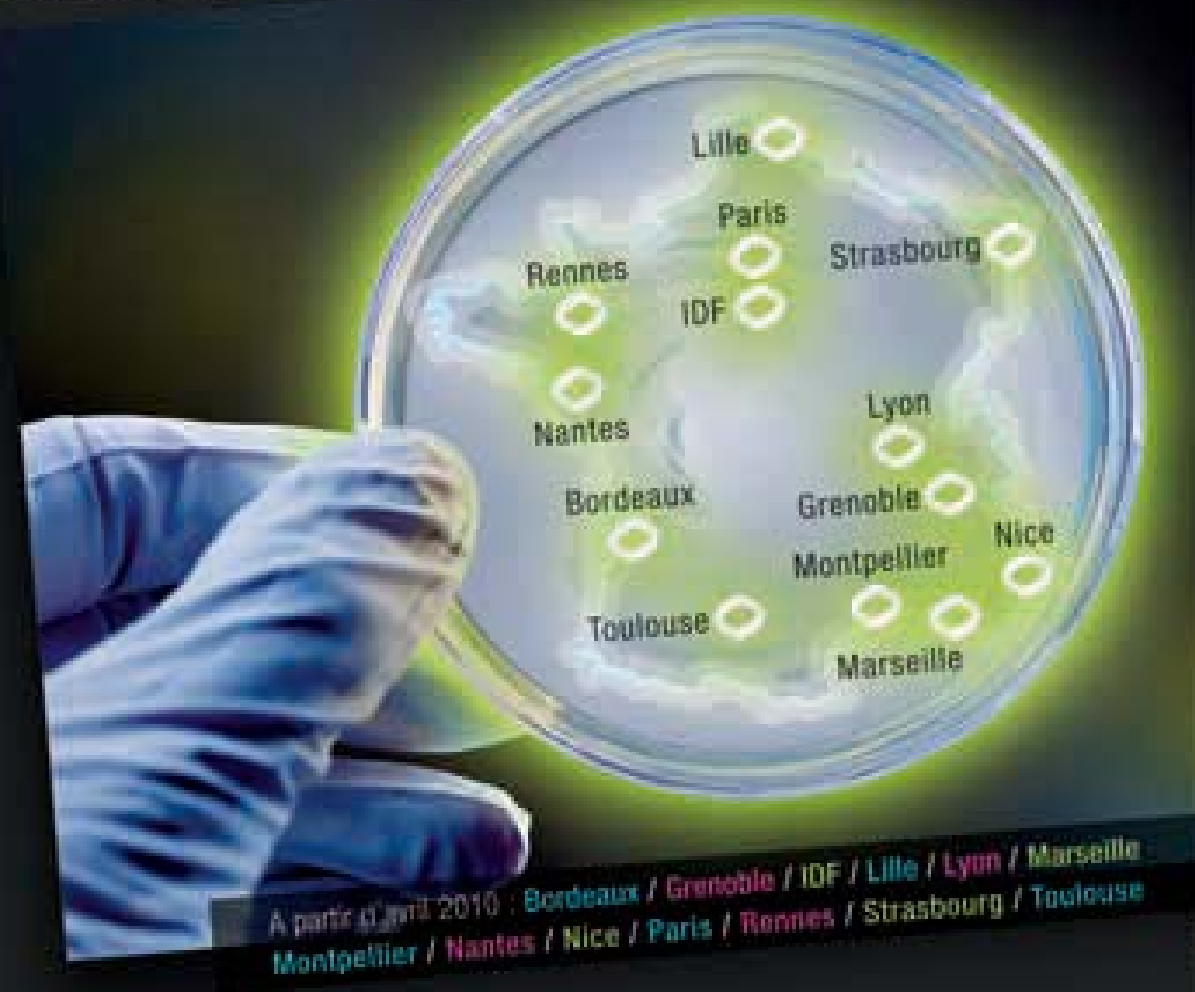

15 Réunions Scientifiques gratuites organisées dans toute la France sur la Bioluminescence et l'Analyse cellulaire in Vivo et in Vitro

Des moments d'echanges et de découvertes entre chercheurs a travers des pretsentations de nombreuses applications in Vivo et in Vitro do ia Lucilerase, Notre objectif : vous pressenter retat oe rart des techiniques en genes rapportecurs, en viabilise cellulaire et en apogtose pour maltriser toutes les possitilités qu'otfrent ces metthodes. Ces rtunions scientflowes sont pratulites of se divelert sur mors drune deni-jante.

N'hésitez pas a vous renseigner augrès de votre dalégue scientifique ou en teliphoriant au 0800487999 ou sur www.promega,com/fr
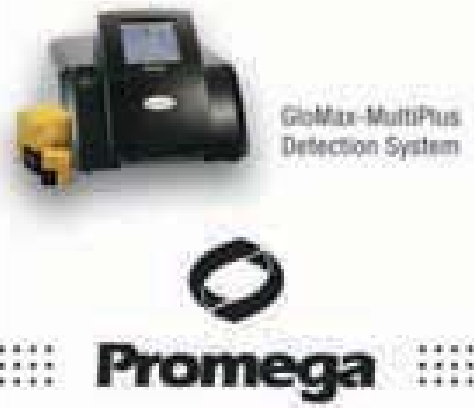